\title{
Erratum to: Evaluation of interferon-gamma release assays for the diagnosis of tuberculosis: an updated meta-analysis
}

\author{
Y. Dai $\cdot$ Y. Feng $\cdot$ R. Xu $\cdot$ W. Xu $・$ W. Lu $\cdot$ J. Wang
}

Received: 14 January 2013 / Accepted: 24 January 2013 /Published online: 27 February 2013

(C) Springer-Verlag Berlin Heidelberg 2013

Erratum to: Eur J Clin Microbiol Infect Dis (2012) 31:3127-3137

\section{DOI 10.1007/s10096-012-1674-y}

Due to some texts delineating the subgroups "T-SPOT.TB" and "QFT" have been unexpectedly swapped around during our editing process, the following changes should be made:

1. Table 1. Exchange the position of the text "QFT (30 studies)" and "T-SPOT (24 studies)".

2. Page 3131. Exchange the position of pooled estimates for QFT and T-SPOT. The correct description should be: "(1) QFT Pooled estimates of the sensitivity, specificity, PLR, NLR, DOR and their $95 \%$ CI were 0.77 (0.75-0.79), $0.81(0.80-0.83), 7.17$ (4.70-10.92), 0.26 (0.20-0.33), 37.24 (19.16-72.40), respectively... (2) T-SPOT Pooled estimates of the sensitivity, specificity, PLR, NLR, DOR and their $95 \%$ CI were 0.89 (0.87-0.91), 0.79 (0.77-0.81), 6.65 (4.28-10.33), 0.14 (0.10-0.20), 61.49 (32.21-117.39), respectively..."

The online version of the original article can be found at http://dx.doi.org/ 10.1007/s10096-012-1674-y.

Y. Dai $\cdot$ Y. Feng $\cdot$ R. Xu $\cdot$ J. Wang $(\bowtie)$

Department of Epidemiology and Biostatistics,

School of Public Health, Nanjing Medical University,

Nanjing 210029, People's Republic of China

e-mail: merry21st@yahoo.com.cn

W. Xu $\cdot$ W. $\mathrm{Lu}$

Department of Chronic Infectious Diseases,

Jiangsu Provincial Center for Disease Prevention and Control,

Nanjing, People's Republic of China 